University of New Orleans

ScholarWorks@UNO

$10-1-1980$

\title{
Mapping of Fresnel's interface reflection coefficients between normal and oblique incidence: results for the parallel and perpendicular polarizations at several angles of incidence
}

\author{
Rasheed M.A. Azzam \\ University of New Orleans, razzam@uno.edu
}

Follow this and additional works at: https://scholarworks.uno.edu/ee_facpubs

Part of the Electrical and Electronics Commons

\section{Recommended Citation}

R. M. A. Azzam, "Mapping of Fresnel's interface reflection coefficients between normal and oblique incidence: results for the parallel and perpendicular polarizations at several angles of incidence," Appl. Opt. 19, 3361-3369 (1980) http://www.opticsinfobase.org/ao/abstract.cfm?URI=ao-19-19-3361

This Article is brought to you for free and open access by the Department of Electrical Engineering at ScholarWorks@UNO. It has been accepted for inclusion in Electrical Engineering Faculty Publications by an authorized administrator of ScholarWorks@UNO. For more information, please contact scholarworks@uno.edu. 


\title{
Mapping of Fresnel's interface reflection coefficients between normal and oblique incidence: results for the parallel and perpendicular polarizations at several angles of incidence
}

\author{
R. M. A. Azzam
}

\begin{abstract}
The functions, $w=f(z)$, that describe the transformation of Fresnel's reflection coefficients of parallel and perpendicularly polarized light between normal and oblique incidence, as well as their inverses, $z=g(w)$, are studied in detail as conformal mappings between the complex $z$ and $w$ planes for angles of incidence of $15,30,45,60$, and $75^{\circ}$. New nomograms are obtained for the determination of optical properties of absorbing isotropic and anisotropic media from measurements of reflectances of $s$ - or $p$-polarized light at normal and oblique incidence.
\end{abstract}

\section{Introduction}

In a recent paper ${ }^{1}$ we have shown that if $w$ and $z$ represent a Fresnel interface reflection or transmission coefficient for a $s$ - (TE) or $p$ - (TM) polarized plane wave of light (or any other electromagnetic radiation) at an oblique angle of incidence $\phi$ and at normal incidence, respectively, $w$ is an analytic function of $z, w=f(z)$, that depends on $\phi$ but not on the media on opposite sides of the interface. We have illustrated in Ref. 1 the mapping between the complex $z$ and $w$ planes for the Fresnel reflection coefficient of the $s$ polarization at one angle of incidence $\left(\phi=45^{\circ}\right)$. In this paper we provide comprehensive documentation of the mapping properties of the reflection coefficients of the $s$ and $p$ polarizations between normal incidence and several angles of oblique incidence $\left(15,30,45,60,75^{\circ}\right)$. Useful nomograms are obtained for determining optical properties of absorbing media from reflectance measurements.

In the following we assume the $\exp (j \omega t)$ time dependence and $p$ and $s$ directions in accordance with the Nebraska (Muller) conventions. ${ }^{2}$

The author is with University of New Orleans, Department of Electrical Engineering, New Orleans, Louisiana 70122.

Received 7 May 1980.

0003-6935/80/193361-09\$00.50/0.

(C) 1980 Optical Society of America.

\section{Reflection Coefficient of the Perpendicular Polarization}

\section{A. Forward Transformation}

The reflection coefficient of $s$-polarized radiation at oblique incidence $w$ is related to that at normal incidence $z$ by ${ }^{1}$

$$
w=\frac{(1-z)-\left(1-a z+z^{2}\right)^{1 / 2}}{(1-z)+\left(1-a z+z^{2}\right)^{1 / 2}},
$$

where

$$
a=4 \tan ^{2} \phi+2 .
$$

In the Nebraska (Muller) conventions, ${ }^{2} z$ and $w$ are limited to the interior and boundaries of the upper half of the unit circle in the complex plane. Figure 1 shows the mapping by Eq. (1) of semicircles centered on the origin of the $z$ plane (representing lines of equal normal-incidence amplitude reflectance $|z|=0.1,0.2, \ldots$, 1) onto the $w$ plane when $\phi=30^{\circ}(a=10 / 3)$. Here, and in all figures, curves and points that are images of one another are denoted by the same numbers and letters, respectively. Points at the beginning (start) and end (finish) of a curve are denoted by $S$ and $F$, and arrows indicate the direction in which curves are traced. Semicircles in the $z$ plane with radii $|z| \leq(1-\sin \phi) /(1$ $+\sin \phi)\left(|z| \leq 1 / 3\right.$ for $\left.\phi=30^{\circ}\right)$ are imaged onto contours in the $w$ plane that begin and end on the real axis (contours, 1, 2, 3, in Fig. 1). For a semicircle with $|z|$ $>(1-\sin \phi) /(1+\sin \phi)$, the image contour in the $w$ plane begins on the unit circle and ends on the real axis. The unit semicircle $|z|=1$ in the $z$ plane is mapped 

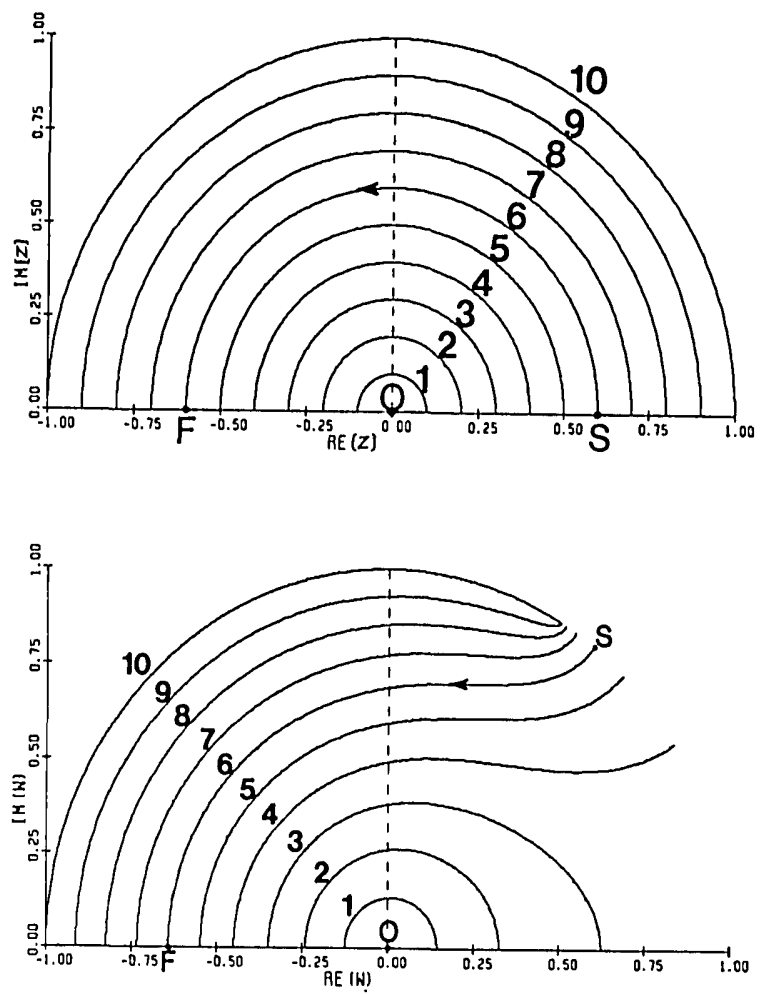

Fig. 1. Mapping of Fresnel's interface reflection coefficient for $s$ polarized light between normal incidence $[z$ plane (top) $]$ and oblique incidence at $30^{\circ}(w$ plane (bottom) $)$. Semicircles $1,2,3, \ldots, 10$ in the $z$ plane are lines of equal normal-incidence amplitude reflectance, $|z|$ $=0.1,0.2,0.3, \ldots, 1$. Images in the $w$ plane carry the same numbers. Start and finish of one semicircle and its image are marked by $S$ and F.
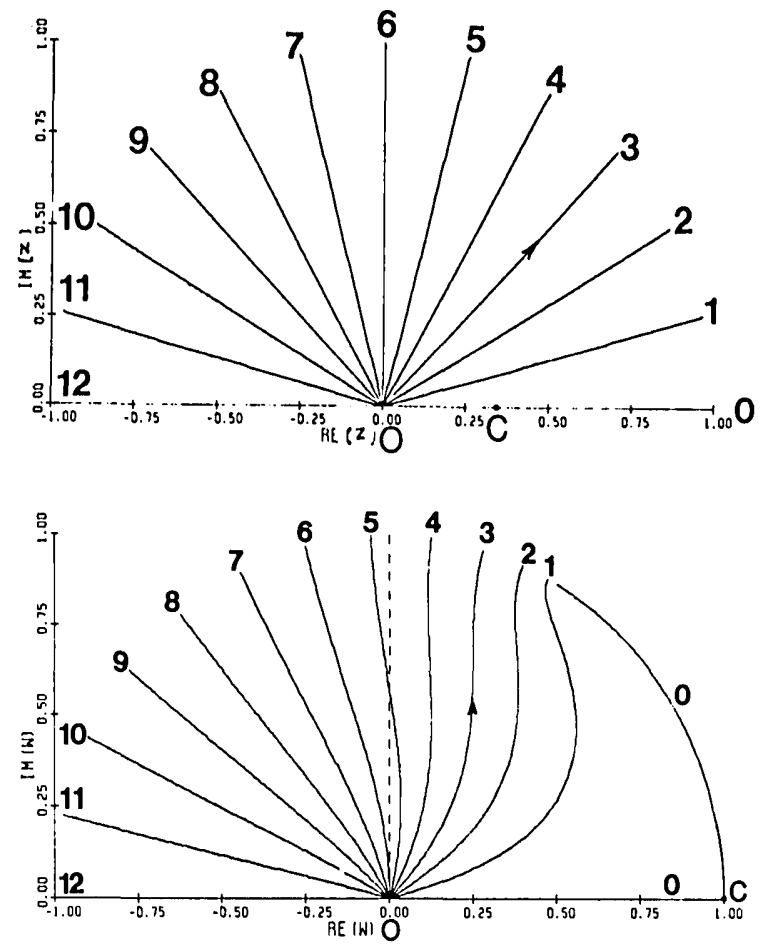

Fig. 2. Same as Fig. 1 for a family of straight lines through the origin $0,1,2, \ldots, 12$ in the $z$ plane, representing lines of equal normal-incidence phase shift, $\arg z=0,15,30, \ldots, 180^{\circ}$. onto the arc of the unit semicircle $|w|=1$ in the $w$ plane between $w=\exp (j 2 \phi)(z=1)$ and $w=\exp (j \pi)(z=-1)$. Points on the unit semicircle $|z|=1$ (other than $z= \pm 1$ ) represent normal-incidence reflection at interfaces between a transparent medium and a medium with a real negative dielectric function (i.e., a plasma).

Figure 2 shows the mapping by Eq. (1) of angularly equispaced straight lines through the origin in the $z$ plane (representing lines of equal normal-incidence reflection phase shift, $\left.\arg z=0,15,30, \ldots, 180^{\circ}\right)$ onto the $w$ plane when $\phi=30^{\circ}(a=10 / 3)$. The origin $O(z=$ $w=0$ ) represents reflection at a vanishing interface (the limiting case when the two surrounding media become the same). Points along the real axis (lines 0 and 12) represent reflection at interfaces between transparent media. When $z=(1-\sin \phi) /(1+\sin \phi)\left(\right.$ point $C, z_{c}=$ $1 / 3$, Fig. 2), we have $w=1$.

The image point $C$ in the $w$ plane $(w=1)$ represents reflection at the critical angle. Thus the segment $0 \leq$ $z \leq z_{c}$ of the real axis of the $z$ plane is mapped onto the segment $0 \leq w \leq 1$ of the real axis of the $w$ plane. Total reflection at $\phi\left(=30^{\circ}\right)$ occurs when $z \geq z_{c}(=1 / 3)$. The segment $z_{c} \leq z \leq 1$ of the real axis of the $z$ plane is mapped onto the arc of the unit circle $|w|=1,0 \leq \arg w$ $\leq 2 \phi$. Notice that the maximum possible total-internal-reflection phase shift for the $s$ polarization at a given angle of incidence $\phi$ is equal to $2 \phi$.

Superposition of Figs. 1 and 2 produces orthogonal sets of curves in the $z$ and $w$ planes, as is expected from a conformal mapping. ${ }^{3}$ This is shown in Fig. 3 for five angles of incidence including $30^{\circ}(\phi=15,30,45,60$, $75^{\circ}$ ).

\section{B. Inverse Transformation}

From Eq. (1), we can solve for $z$ in terms of $w$; this gives

$$
z=\frac{(a+2 p) \pm\left[\left(a^{2}-4\right)+4(a+2) p\right]^{1 / 2}}{2(1-p)},
$$

where

$$
p=[(1-w) /(1+w)]^{2},
$$

and $a$ is defined by Eq. (2).

The inverse mapping by Eq. (3) of semicircles centered on, and straight lines through, the origin in the $w$ plane onto the $z$ plane is illustrated by Figs. 4 and 5, respectively, for $\phi=30^{\circ}(a=1 \%)$.

The superposition of Figs. 4 and 5 gives orthogonal sets of curves in the $w$ and $z$ planes. This is shown in Fig. 6 for five angles of incidence, including $30^{\circ}(\phi=15$, $\left.30,45,60,75^{\circ}\right)$. 


\section{Reflection Coefficient of the Parallel Polarization}

A. Forward Transformation

The reflection coefficients of $p$-polarized radiation at oblique incidence $w$ and at normal incidence $z$ are interrelated by ${ }^{1}$

$$
w=\frac{(1+z)^{2}-(1-z)\left(1+a z+z^{2}\right)^{1 / 2}}{(1+z)^{2}+(1-z)\left(1+a z+z^{2}\right)^{1 / 2}}
$$

where $a$ is defined by Eq. (2).

In the Nebraska (Muller) conventions, ${ }^{2} z$ is limited to the inside and boundaries of the lower half of the unit circle in the complex plane, and $w$ is confined to the interior and boundary of the full unit circle.
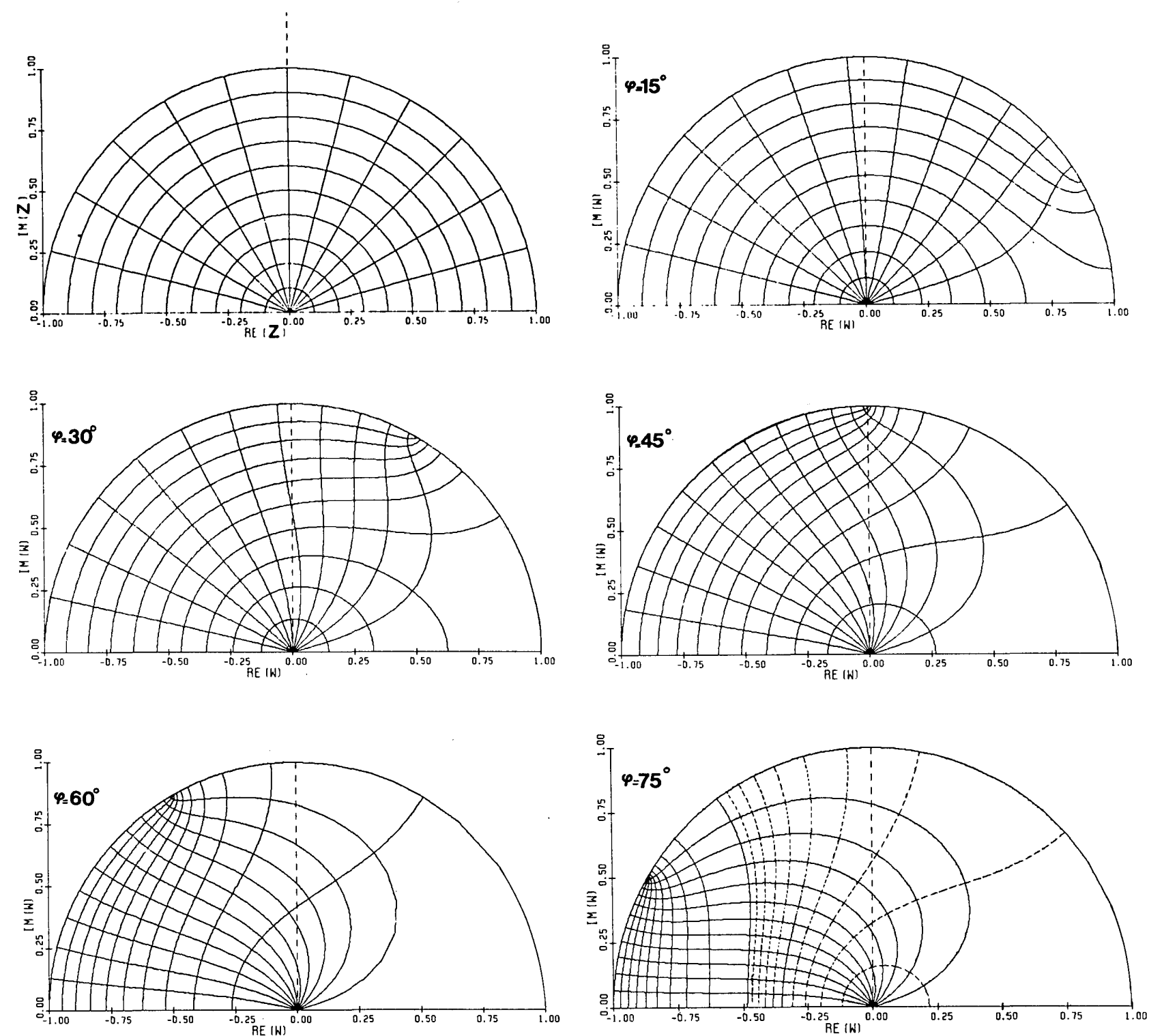

Fig. 3. Superposition of Figs. 1 and 2 gives orthogonal sets of curves in the $z$ and $w$ planes. Results shown are for five angles of incidence including $30^{\circ}\left(\phi=15,30,45,60,75^{\circ}\right)$. To identify individual curves, use Figs. 1 and $2\left(\phi=30^{\circ}\right)$ as a guide. Dashed curves in the graph of $\phi=75^{\circ}$ are images of semicircles $|z|=0.01 m$, where $m=1,2, \ldots, 9$.
Figure 7 shows the mapping by Eq. (5) of semicircles centered on the origin of the complex $z$ plane (representing lines of equal normal-incidence amplitude reflectance $|z|=0.1,0.2, \ldots, 1.0)$ onto the $w$ plane when the angle of incidence $\phi$ is $30^{\circ}(a=10 \%)$. The notation is the same as discussed Sec. II.

Two points on the real axis of the $z$ plane have special significance to the reflection of $p$-polarized light. Point $B$ with $z=\tan \left(\phi-45^{\circ}\right)\left(=-0.268\right.$ for $\left.\phi=30^{\circ}\right)$ is mapped onto the origin, $w=0$, of the $w$ plane, corresponding to wave reflection at the Brewster angle. Point $M, z=(\sqrt{2} \sin \phi-1) /(\sqrt{2} \sin \phi+1)(=-0.1715$ for $\left.\phi=30^{\circ}\right)$, is mapped onto $w=-\tan ^{2}\left(\phi-45^{\circ}\right)(=$ 

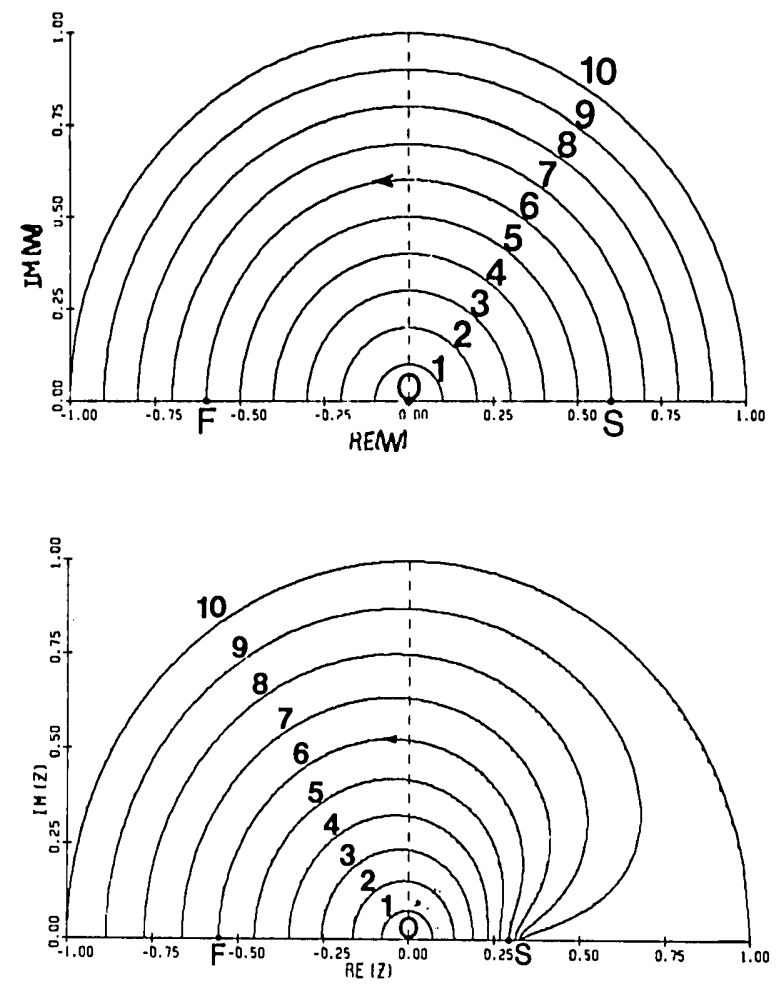

Fig. 4. Inverse mapping of Fresnel's interface reflection coefficient for $s$-polarized light between oblique incidence at $30^{\circ}$ [ $\mathrm{w}$ plane (top)] and normal incidence $[z$ plane (bottom)]. Semicircles $1,2,3, \ldots, 10$ in the $w$ plane are lines of constant oblique-incidence $\left(30^{\circ}\right)$ amplitude reflectance $|w|=0.1,0.2,0.3, \ldots, 1$. Their images in the $z$ plane are marked by the same numbers.
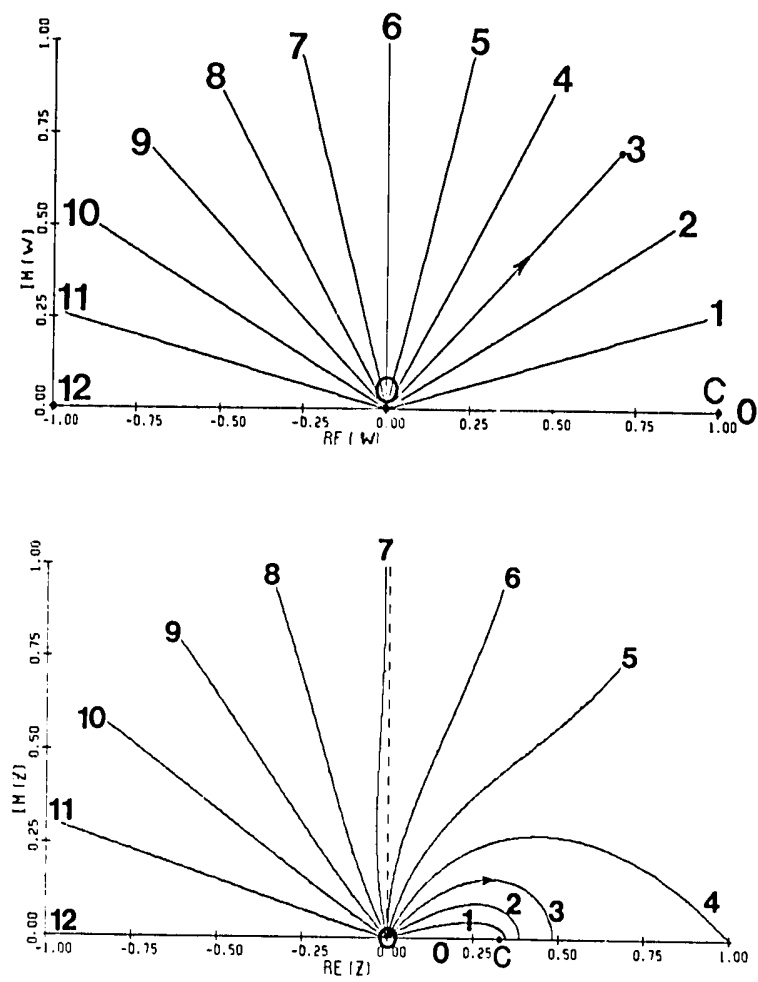

Fig. 5. Same as in Fig. 4 for a family of straight lines through the origin $0,1,2, \ldots, 12$ in the $w$ plane representing lines of constant oblique-incidence $\left(30^{\circ}\right)$ phase shift, $\arg w=0,15,30, \ldots, 180^{\circ}$.
-0.0718 for $\left.\phi=30^{\circ}\right)$, corresponding to wave refraction at $45^{\circ} .4$ Point $C, z=(\sin \phi-1) /(\sin \phi+1)(=-0.333$ for $\phi=30^{\circ}$ ) is mapped onto $w=1$, representing wave reflection at the critical angle $\left(90^{\circ}\right.$ refraction). Semicircles with radii $|z|<|O M|$ (semicircle 1 in Fig. 7) are mapped onto contours that lie entirely in the lower half of the $w$ plane. Semicircles with radii in the range $|O M|<|z|<|O C|$ (2 and 3 in Fig. 7) are mapped onto one-full-revolution spirals in the $w$ plane beginning and ending on the real axis. The spiraling is around the point $M$ when $|z|$ is only slightly greater than $|O M|$. Images of semicircles with radii $|z|>|O C|$ (4-10 in Fig. 7) resemble spirals of less than one full revolution, each of which begins on the upper half of the unit circle in the $w$ plane and ends on the real axis.

Figure 8 shows the mapping by Eq. (5) of angularly equispaced straight lines through the origin in the $z$ plane (representing lines of equal normal-incidence reflection phase shift, $\arg z=180,195,210, \ldots, 360^{\circ}$ ) onto the $w$ plane for $\phi=30^{\circ}(a=10 / 3)$. Of particular interest is the mapping of line $0\left(\arg z=180^{\circ}\right)$. As $z$ moves along the negative real axis from $O$ to $M$ to $B, w$ moves along the negative real axis of the $w$ plane first leftward from the origin to $M$, then reverses direction at $M$ and moves rightward from $M$ back to the origin $(O, B)$. Subsequent motion of $z$ from $B$ to $C$ is mirrored into motion of $w$ from the origin $O$ to $C(w=1)$. Finally, the image of the remaining segment of line 0 between $C$ and $z=-1$ in the $z$ plane is the upper half of the unit circle in the $w$ plane. The reversal of the direction of motion of $w$ at $M$ is an expression of the stationary property of $w$ at that point, ${ }^{4}$ namely, $\partial w / \partial z=$ 0 .

Superposition of Figs. 7 and 8 produces orthogonal sets of curves in the $z$ and $w$ planes, as is expected from a conformal mapping. This is shown in Fig. 9 for five angles of incidence including $30^{\circ}(\phi=15,30,45,60$, $\left.75^{\circ}\right)$.

\section{B. Inverse Transformation}

In Ref. 1 we showed that to determine the complex $p$-reflection coefficient at normal incidence $z$ from the value that this coefficient assumes at some angle of oblique incidence $w$, Eq. (5) is solved for $z$ in the terms of $w$. This gave rise to a quartic (fourth-degree) algebraic equation in $z$ with coefficients that are functions of $w$ and the angle of incidence. Instead of solving such a quartic equation, and deciding on the proper root, we have found two other alternative methods that are simple, direct, and explicit.

The first makes use of the recently obtained direct relation between Fresnel's reflection coefficients for the $p$ and $s$ polarizations at the same angle of incidence $\phi$. If $w_{p}$ and $w_{s}$ denote these coefficients, ${ }^{5}$

$$
\begin{aligned}
& w_{p}=w_{s}\left(w_{s}-\cos 2 \phi\right) /\left(1-w_{s} \cos 2 \phi\right), \\
& w_{s}=1 / 2 \cos 2 \phi\left(1-w_{p}\right) \pm\left[w_{p}+1 / 4 \cos ^{2} 2 \phi\left(1-w_{p}\right)^{2}\right]^{1 / 2} .
\end{aligned}
$$

For a given $w_{p}$ at a given $\phi$, we compute $w_{s}$ from Eq. (6b), selecting the root with $\operatorname{Im} w_{s}>0$. Next we compute $z_{s}$ from $w_{s}$ using Eqs. (3) and (4) and select the root with $\operatorname{Im} z_{s}>0$. Finally, $z_{p}$ is obtained as $-z_{s}$. 
In the second method, we start with the basic equation for the Fresnel coefficient of the $p$ polarization ${ }^{6}$ :

$$
w_{p}=\frac{\epsilon \cos \phi-\left(\epsilon-\sin ^{2} \phi\right)^{1 / 2}}{\epsilon \cos \phi+\left(\epsilon-\sin ^{2} \phi\right)^{1 / 2}},
$$

where $\epsilon$ is the ratio of the complex dielectric constants of the media of refraction and incidence. We solve Eq. (7) for $\epsilon$ :

$$
\epsilon=\left[1 \pm\left(1-Q_{p} \sin ^{2} 2 \phi\right)^{1 / 2}\right] / 2 Q_{p} \cos ^{2} \phi,
$$

where
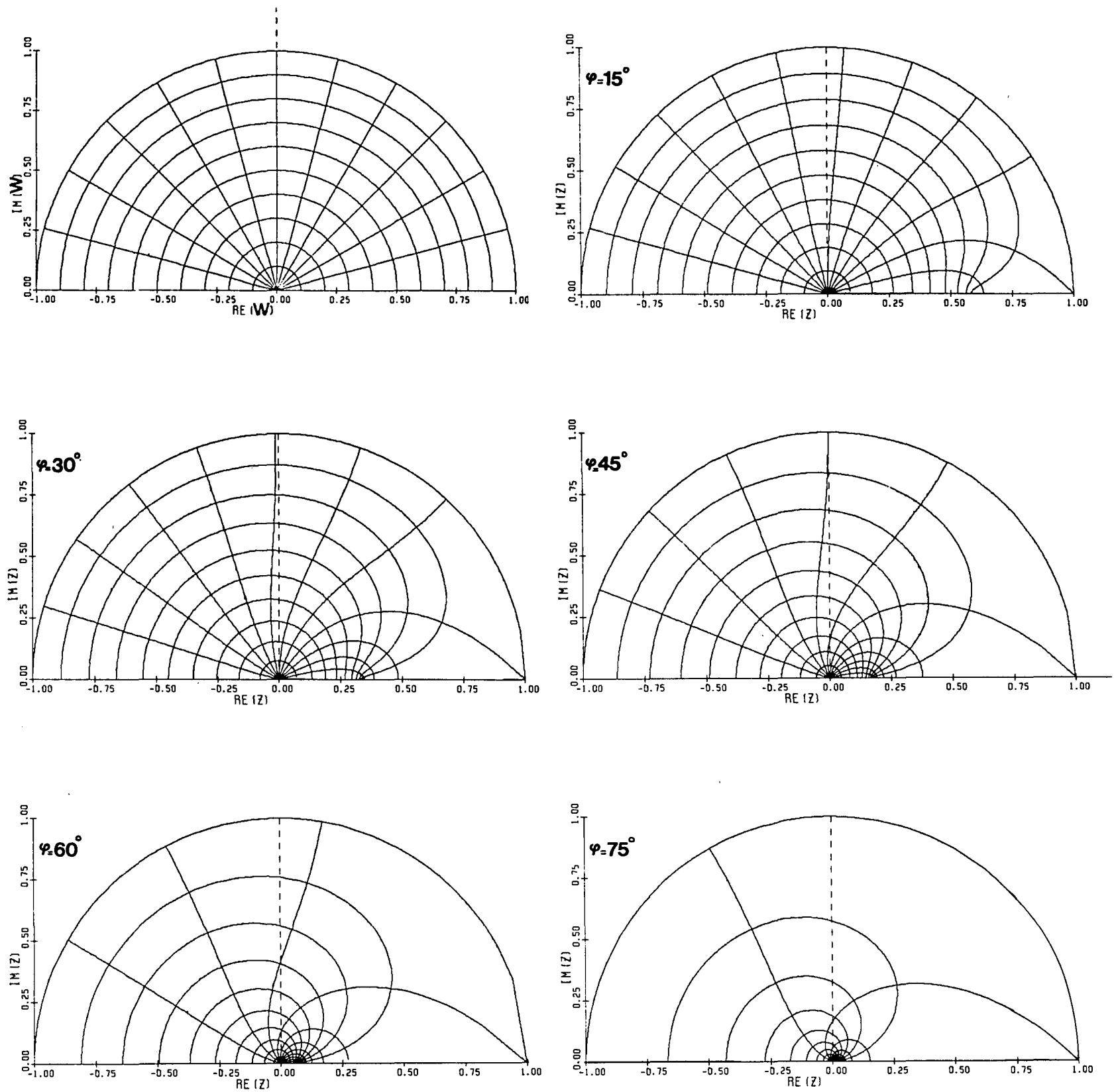

Fig. 6. Superposition of Figs. 4 and 5 produces orthogonal families of curves in the $w$ and $z$ planes. Results shown are for five angles of incidence including $30^{\circ}\left(\phi=15,30,45,60,75^{\circ}\right)$. To identify individual curves use Figs. 4 and 5 (for $\left.\phi=30^{\circ}\right)$ as a guide. 

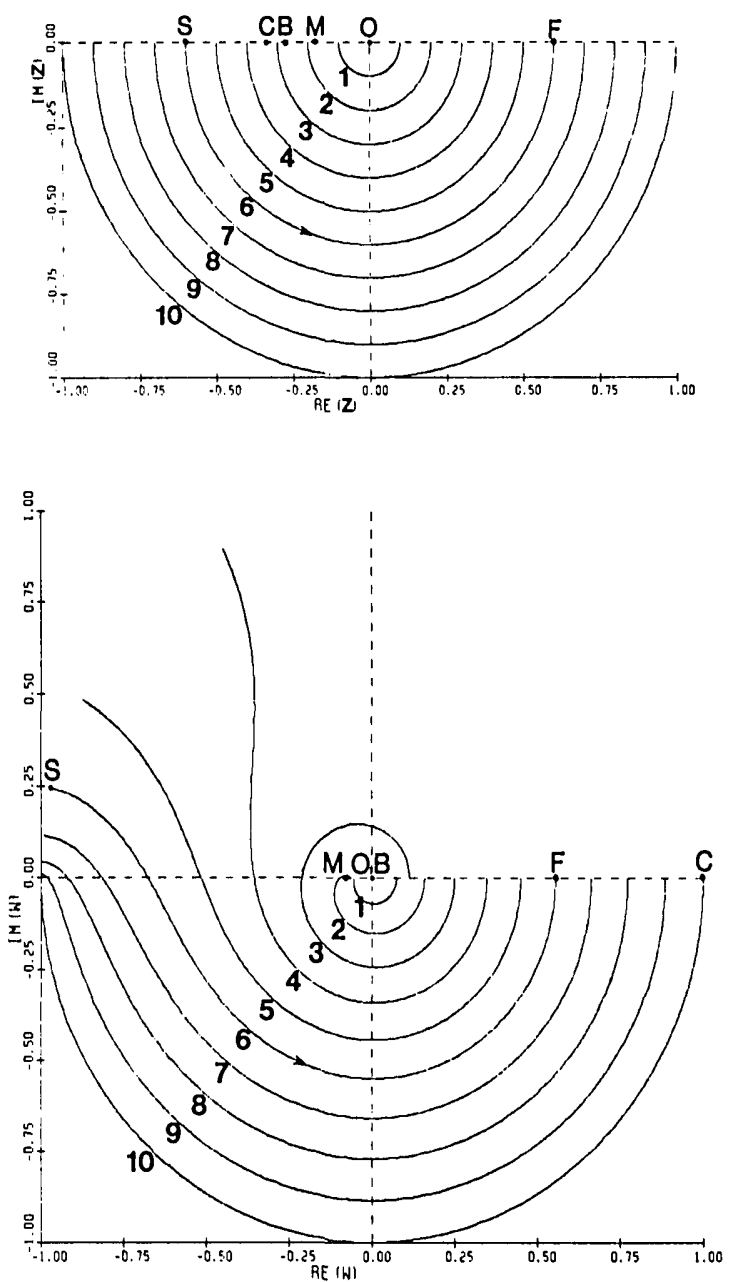

Fig. 7. Mapping of Fresnel's interface reflection coefficient for $p$ polarized light between normal incidence $[z$ plane (top) $]$ and oblique incidence at $30^{\circ}[w$ plane (bottom) $]$. Semicircles $1,2,3, \ldots, 10$ in the $z$ plane are lines of equal normal-incidence amplitude reflectance, $|z|$ $=0.1,0.2,0.3, \ldots, 1$. Their images in the $w$ plane are marked by the same numbers. Significance of points $B, C$, and $M$ is discussed in the text.

above to study the inverse $w \rightarrow z$ mapping of the reflection coefficient for the parallel polarization. Figure 10 shows the mapping of concentric circles centered on the origin of the $w$ plane (representing lines of equal amplitude $p$ reflectance at $\phi=30^{\circ},|w|,=0.1,0.2,0.3$, $\ldots, 1.0)$ onto the $z$ plane. All image contours in the $z$ plane start on the short segment of the real axis between $B\left[z=\tan \left(\phi-45^{\circ}\right)=-0.268\right]$ and $C[z=(\sin \phi-1) /$ $(\sin \phi+1)=-0.333]$ and end on the segment of the real axis between $O$ and $z=+1$. The letter and arrow notations are the same as explained in Sec. II.

Figure 11 shows the mapping of angularly equispaced straight lines through the origin of the $w$ plane (lines of equal $p$-reflection phase shift, $\arg w=0,15,30, \ldots, 360^{\circ}$ at $\phi=30^{\circ}$ ) onto the $z$ plane. The origin of the $w$ plane has separate double images $O$ and $B$ in the $z$ plane. Lines 1-11 in the upper half of the $w$ plane are imaged onto a group of curves that all emanate from $B(z=$ -0.268 ) and end on the segment of the real axis between
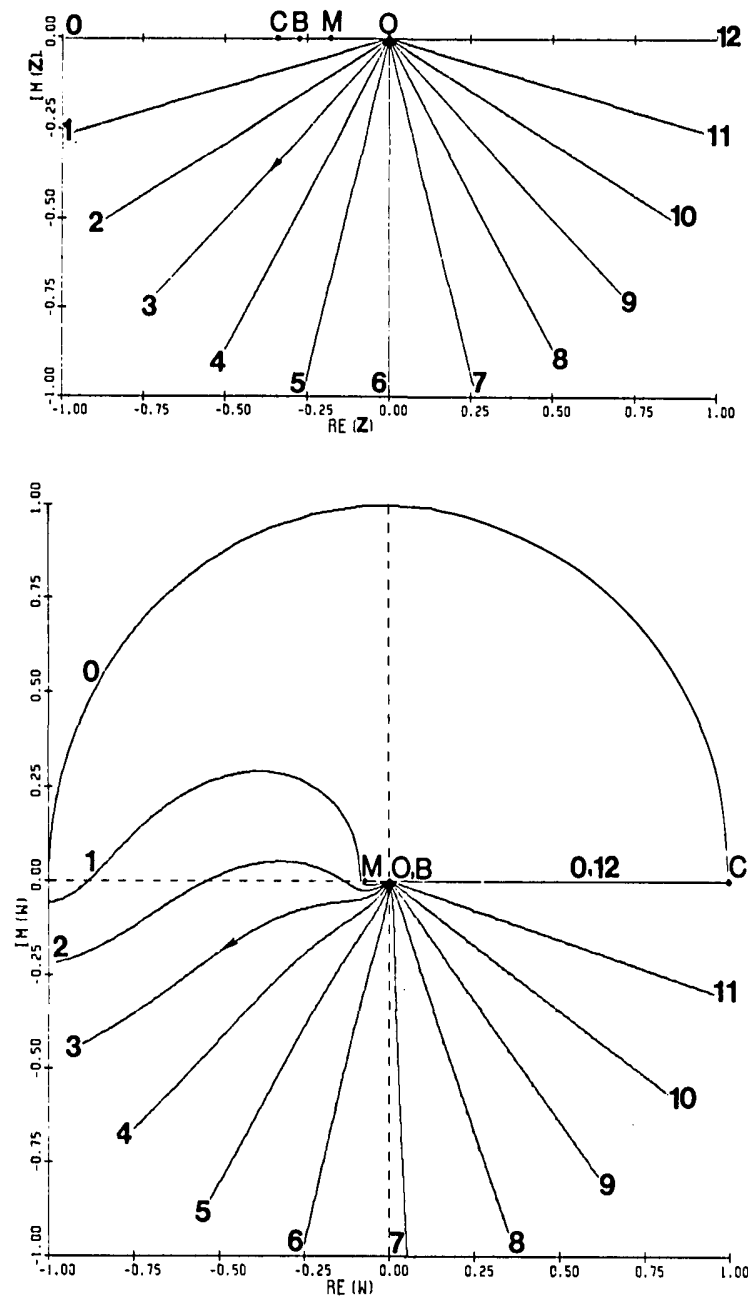

Fig. 8. Same as in Fig. 7 for a family of straight lines through the origin $0,1,2, \ldots, 12$ in the $z$ plane, representing lines of equal normal-incidence phase shift, $\arg z=180,195,210, \ldots, 360^{\circ}$.

$C(z=-0.333)$ and $z=-1$. Lines $13-23$ in the lower half of the $w$ plane are imaged onto a separate family of curves that all eminate from the origin $O$ and terminate on the circumference of the lower half of the unit circle in the $z$ plane. The inverse transformation is doublevalued for real values of $w$ represented by points on the segment of the real axis of the $w$ plane between $M[w=$ $\left.-\tan ^{2}\left(\phi-45^{\circ}\right)=-0.0718\right]$ and $C(w=1)$. The segment from the origin to $M$ in the $w$ plane is imaged onto two separate segments of the real axis of the $z$ plane from $O$ to $M$ and from $B$ and $M$. The segment from $w=0$ to $w=1$ also has double images from $B$ to $C$ and from $O$ to $z=1$.

The superposition of Figs. 10 and 11 generates orthogonal families of curves in the $w$ and $z$ planes, as is expected from a conformal mapping. This is shown in Fig. 12 for five angles of incidence including $30^{\circ}(\phi=15$, $\left.30,45,60,75^{\circ}\right)$. 

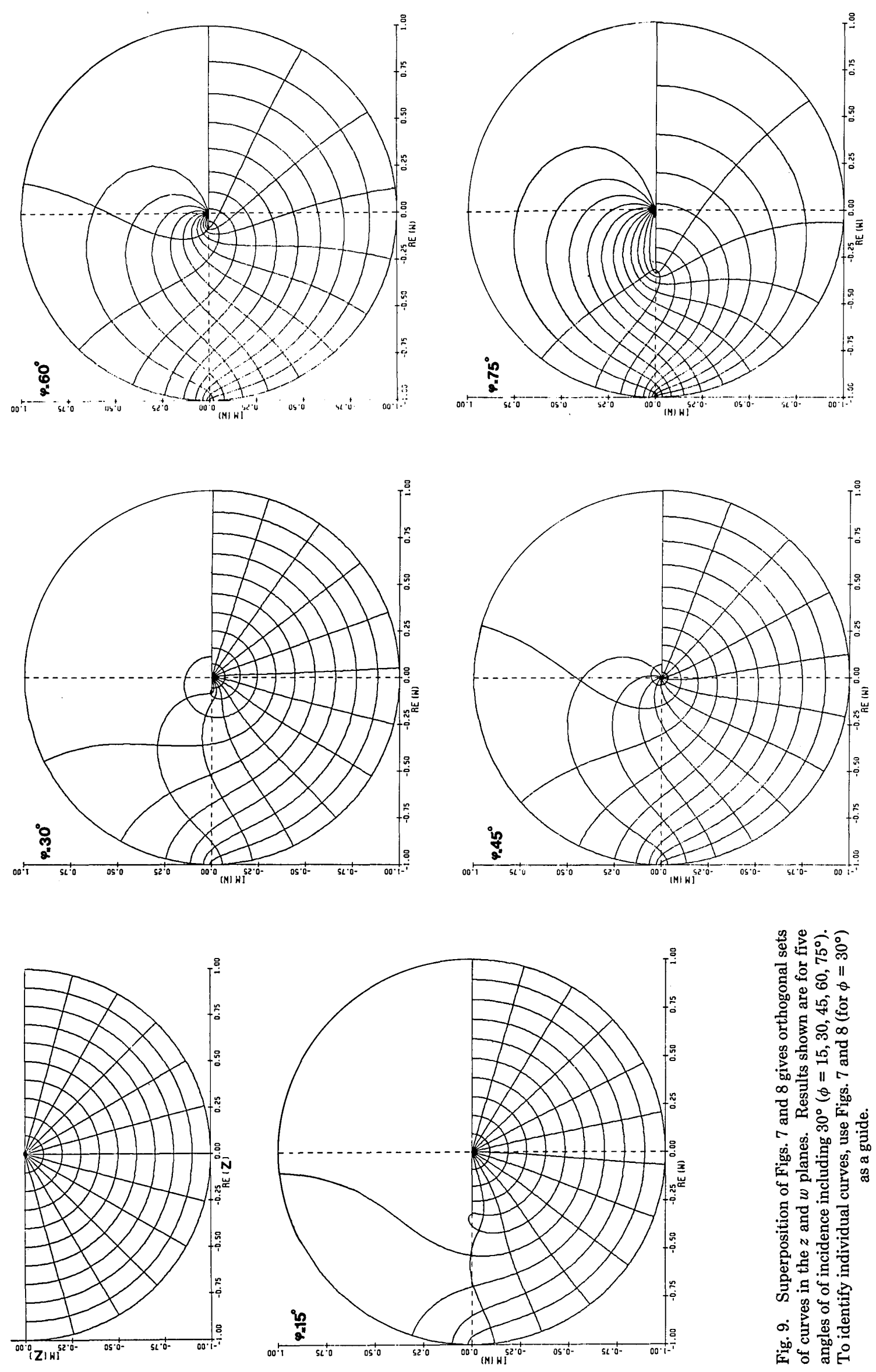

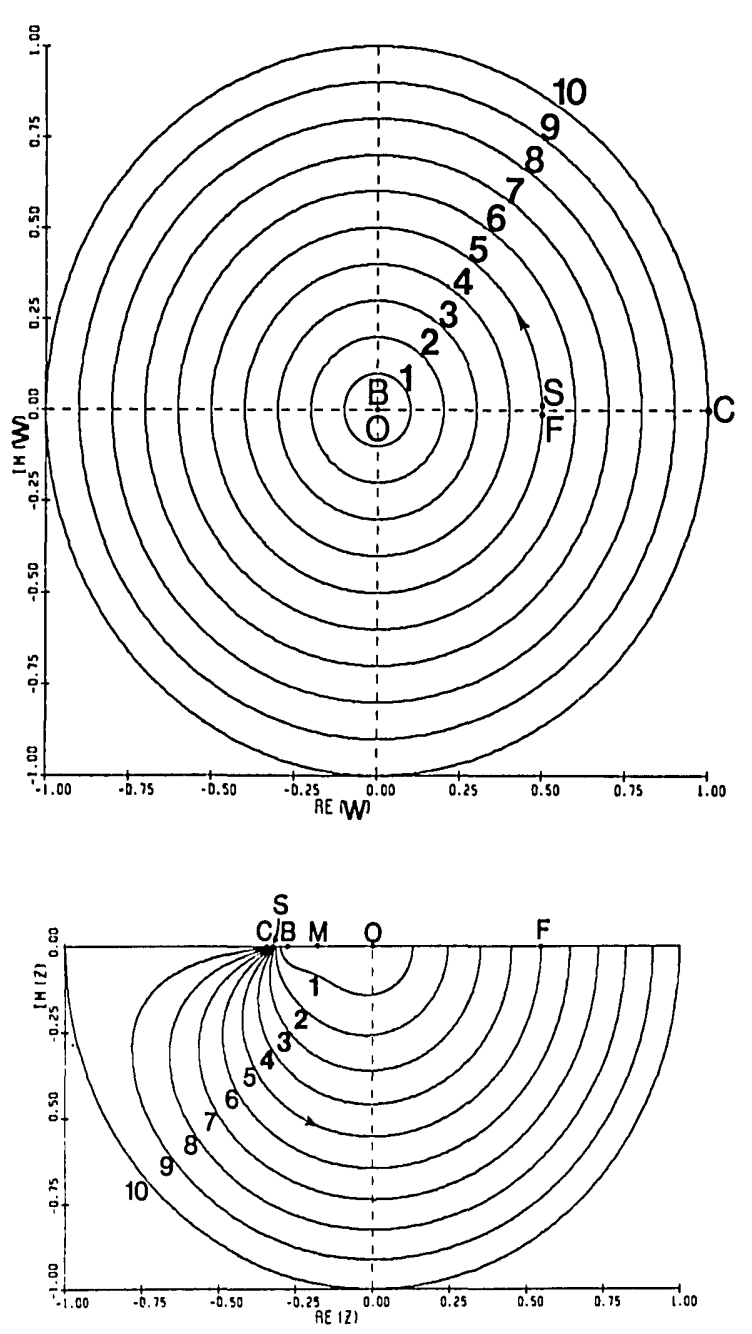

Fig. 10. Inverse mapping of Fresnel's interface reflection coefficient for $p$-polarized light between oblique incidence at $30^{\circ}$ [ $w$ plane (top)] and normal incidence $[z$ plane (bottom)]. Full circles centered on the origin $1,2,3, \ldots, 10$ in the $w$ plane are lines of constant obliqueincidence $\left(30^{\circ}\right)$ amplitude reflectance, $|w|=0.1,0.2,0.3, \ldots, 1$. Their images in the $z$ plane are marked by the same numbers. Significance of points $B, C$, and $M$ is discussed in the text.

\section{Determination of Optical Properties from Measured Reflectances at Normal and Oblique Incidence}

Let $s$ - or $p$-polarized light be incident from a transparent isotropic ambient of known refractive index (e.g., air) onto the planar surface of an isotropic absorbing medium of unknown complex refractive index. Measurements of the intensity reflectances at normal incidence $R_{0}$ and at oblique incidence $R_{\phi}$ determine $|z|=$ $R_{0}^{1 / 2}$ and $|w|=R_{\phi}^{1 / 2}$. The intersection in the complex $z$ plane of the circle $|z|=R_{0}^{1 / 2}$ with the image of $|w|=$ $R_{\phi}^{1 / 2}$ specifies the complex normal-incidence reflection coefficient $z$. Hence

$$
N= \pm n(1-z) /(1+z),
$$

where + is for the $s$ polarization, and - is for the $p$ polarization.

In Ref. 1, we have illustrated how this technique works for $s$-polarized light and assuming reflection at normal and $45^{\circ}$ incidence and provided one nomogram appropriate to this case. The extension of this tworeflectance method (TRM) to the determination of the optical properties of uniaxial and biaxial absorbing crystals was also discussed. The results of this paper, in particular Fig. 6, make available additional nomograms that permit the use of the TRM at other angles of incidence of $15,30,60$, and $75^{\circ}$. The nomograms are simply realized by superimposing on Fig. 6 a transparency with concentric circles of equispaced radii that represent lines of $|z|=$ constant. Contours of $\arg w=$ constant can be either ignored or deleted. Because we adhere to the Nebraska (Muller) conventions, ${ }^{2}$ the present nomograms are one-half of the size used in Ref. 1.

Figure 12, and a transparency of concentric circles, provide useful nomograms for applying the TRM with $p$-polarized light, thus complementing the results of Ref. 1. This is of particular importance in view of the acknowledged higher sensitivity of TRM using the $p$ polarization, as compared to the $s$ polarization, ${ }^{7}$ for determining the optical properties of materials. However, extension of our TRM using $p$-polarized light to anisotropic media is not as straightforward as possible with $s$-polarized light.
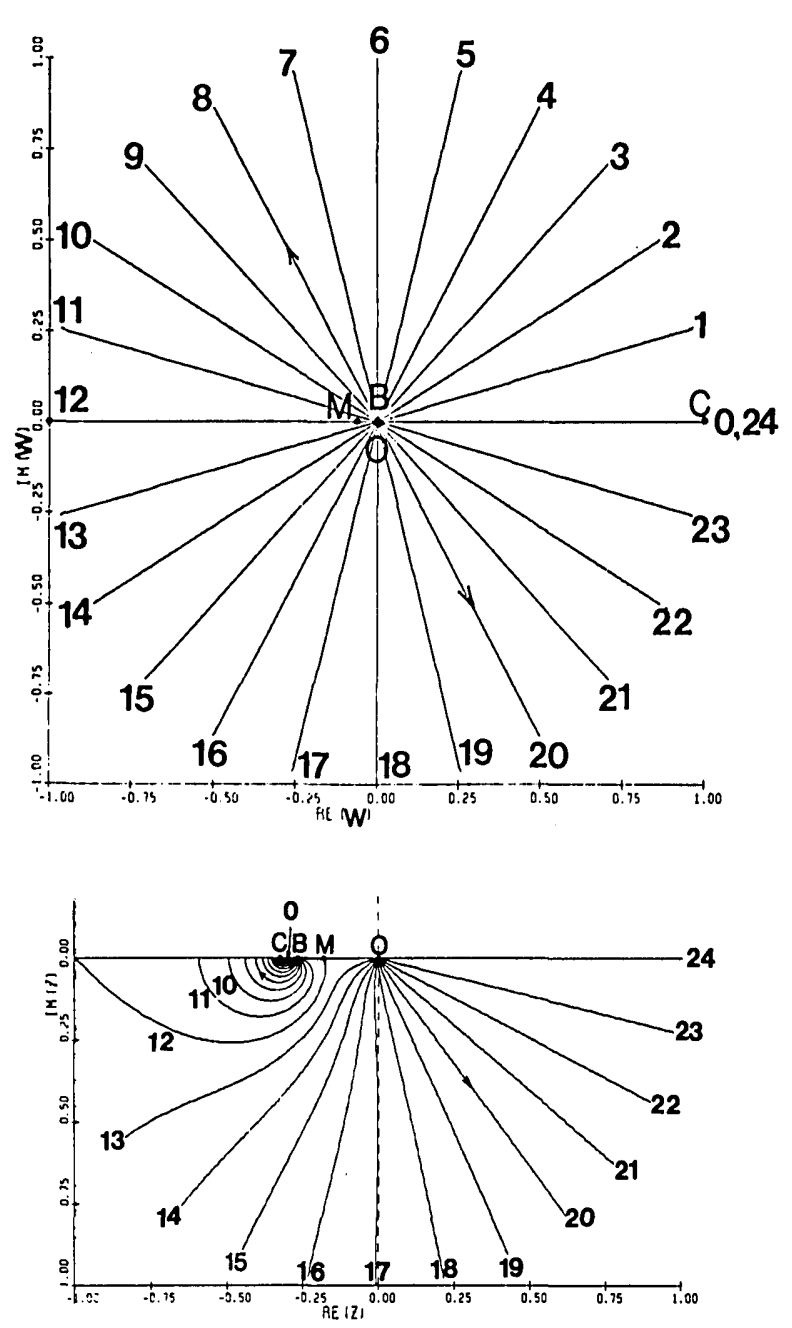

Fig. 11. Same as in Fig. 10 for a family of straight lines through the origin $0,1,2, \ldots, 24$ in the $w$ plane (top) representing lines of constant oblique-incidence $\left(30^{\circ}\right)$ phase shift, $\arg w=0,15,30, \ldots, 360^{\circ}$. 

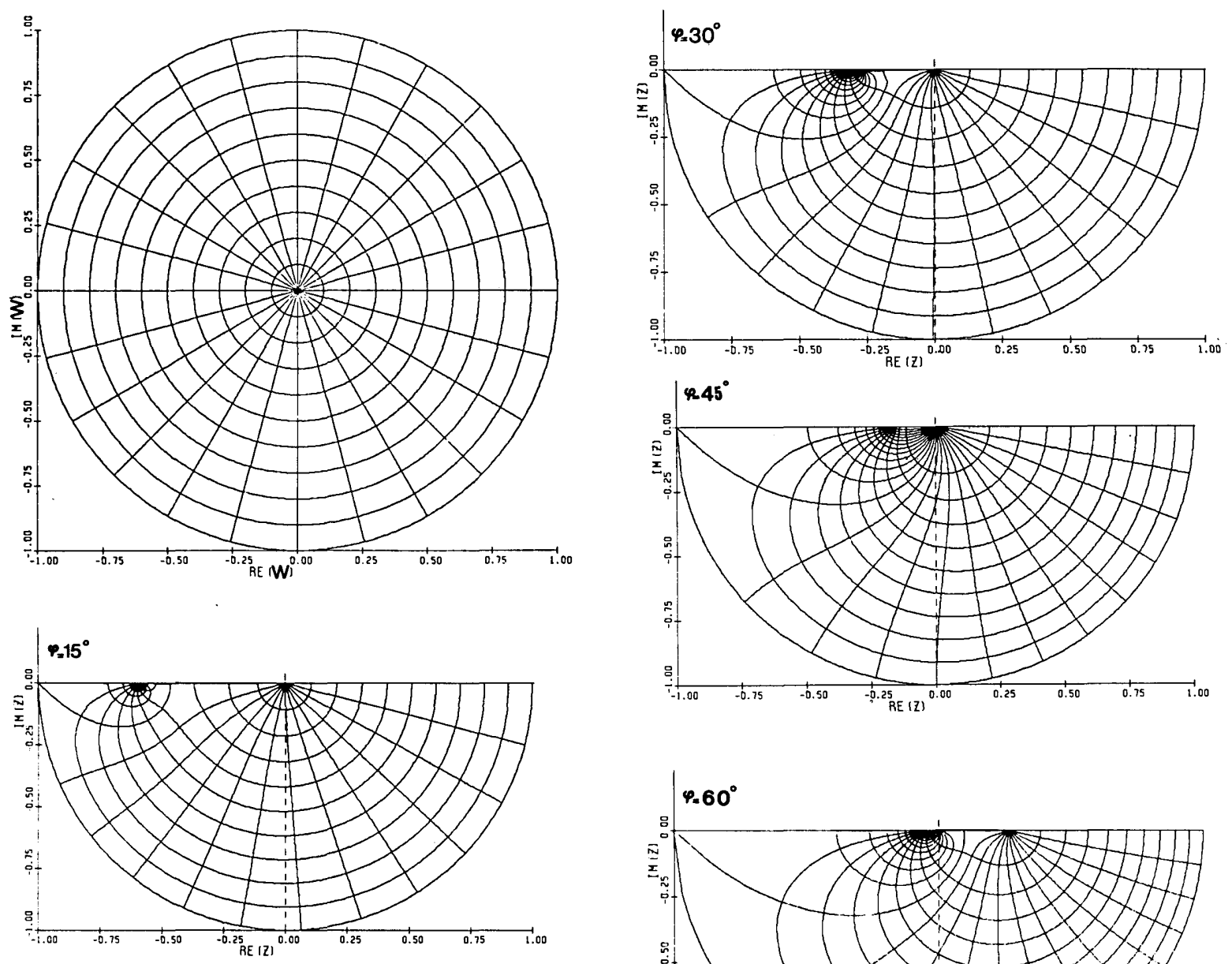

Fig. 12. Superposition of Figs. 10 and 11 gives orthogonal sets of curves in the $w$ and $z$ planes. Results shown are for five angles of incidence including $30^{\circ}\left(\phi=15,30,45,60,75^{\circ}\right)$. To identify individual curves use Figs. 10 and 11 as a guide.

\section{Conclusion}

The present paper complements our earlier work ${ }^{1}$ and gives detailed documentation of how the basic Fresnel reflection coefficients are transformed between normal and oblique incidence. Only the case of $s$-polarized radiation and reflection at the special angle of $45^{\circ}$ was illustrated before. Here we have removed this limitation by considering both the $s$ and $p$ polarizations and reflection at several $\left(15,30,45,60,70^{\circ}\right)$ angles of incidence. Families of curves thus obtained provide useful nomograms for applying the TRM to the determination of optical properties of isotropic and anisotropic absorbing media. The case of the $p$ polarization, considered here for the first time, is especially important in this application because of the higher sensitivity that the TRM attains with its use.
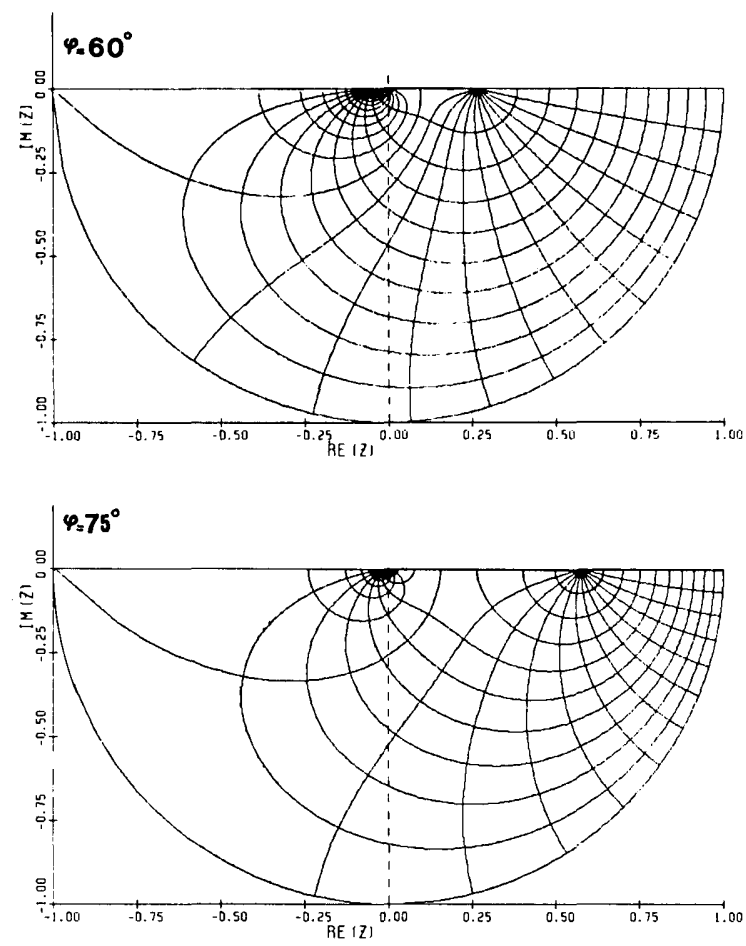

\section{References}

1. R. M. A. Azzam, J. Opt. Soc. Am. 69, 590 (1979).

2. R. H. Muller, Surf. Sci. 16, 14 (1969).

3. See, for example, A. Kyrala, Applied Functions of a Complex Variable (Wiley-Interscience, New York, 1972), Chap. 8.

4. R. M. A. Azzam, J. Opt. Soc. Am. 68, 1613 (1978).

5. R. M. A. Azzam, J. Opt. Soc. Am. 69, 1007 (1979).

6. See, for example, M. Born and E. Wolf, Principles of Optics (Pergamon, New York, 1975), p. 40.

7. W. R. Hunter, J. Opt. Soc. Am. 55, 1197 (1965). 